\title{
Numerical study of roughness distributions in nonlinear models of interface growth
}

\author{
F. D. A. Aarão Reis \\ Instituto de Física, Universidade Federal Fluminense, \\ Avenida Litorânea s/n, 24210-340 Niterói RJ, Brazil
}

(Dated: May 12, 2019)

\begin{abstract}
We analyze the shapes of roughness distributions of discrete models in the Kardar, Parisi and Zhang (KPZ) and in the Villain, Lai and Das Sarma (VLDS) classes of interface growth, in one and two dimensions. Three KPZ models in $d=2$ confirm the expected scaling of the distribution and show a stretched exponential tail approximately as $\exp \left(-x^{0.8}\right)$, with a significant asymmetry near the maximum. Conserved restricted solid-on-solid models belonging to the VLDS class were simulated in $d=1$ and $d=2$. The tail in $d=1$ has the form $\exp \left(-x^{2}\right)$ and, in $d=2$, has a simple exponential decay, but is quantitatively different from the distribution of the linear fourth-order (Mullins-Herring) theory. It is not possible to fit any of the above distributions to those of $1 / f^{\alpha}$ noise interfaces, in contrast with recently studied models with depinning transitions.
\end{abstract}

PACS numbers: PACS numbers: 05.40.-a, 05.50.+q, 68.55.-a, 81.15.Aa 


\section{INTRODUCTION}

The simplest quantitative characteristic of an interface is its width, or roughness, $w$, defined as the rms fluctuation of the height around its average position. The scaling of the average width of different realizations with the length $L$ and the time $t$ is useful for analyzing interfaces formed in several processes [1, 2]. Another potentially useful characteristic is the full roughness distribution at the steady state, particularly in cases where reliable estimates of scaling exponents are not available.

The distributions for linear growth models, such as the Edwards-Wilkinson (EW) and the Mullins-Herring ( $\mathrm{MH})$ equations, were obtained exactly in $d=1$ and can be computed with high degree of accuracy in $d=2[3,4,5]$. The generating functions for Gaussian interfaces with power spectrum of the type $1 / f^{\alpha}$ were computed by the same methods [6], 7]. However, many real interfaces are described by nonlinear equations at a coarse-grained level, and their scaling exponents and roughness distributions are exactly known only in some particular cases. Consequently, their accurate numerical calculation may provide a basis for comparison with results from experiments or model systems. In this Brief Report, we will study numerically the distributions of models governed by second and fourth order nonlinear growth equations, in $d=1$ and $d=2$.

The first model is that of Kardar, Parisi and Zhang (KPZ) [8], in which the local height $h$ at position $\vec{x}$ and time $t$ evolves as

$$
\frac{\partial h}{\partial t}=\nu_{2} \nabla^{2} h+\lambda_{2}(\nabla h)^{2}+\eta(\vec{x}, t)
$$

Here, $\nu_{2}$ and $\lambda_{2}$ are constants and $\eta$ is a Gaussian noise with zero mean and variance $\left\langle\eta(\vec{x}, t) \eta\left(\overrightarrow{x^{\prime}}, t^{\prime}\right)\right\rangle=D \delta^{d}\left(\vec{x}-\overrightarrow{x^{\prime}}\right) \delta\left(t-t^{\prime}\right)$, where $d$ is the dimension of the substrate. In the case $\lambda_{2}=0$, we obtain the EW equation.

Another important nonlinear model is that of Villain [9] and Lai and Das Sarma 10], the so-called VLDS equation (or nonlinear MBE equation)

$$
\frac{\partial h}{\partial t}=\nu_{4} \nabla^{4} h+\lambda_{4} \nabla^{2}(\nabla h)^{2}+\eta(\vec{x}, t)
$$

where $\nu_{4}$ and $\lambda_{4}$ are constants. The linear version of Eq. (2) $\left(\lambda_{4}=0\right)$ is the MH equation 1 , 4, 5, 11, 12].

Previous studies [3, 5] of the roughness distributions of discrete models in these classes 
suggested that they have a universal shape, with the form

$$
P_{L}\left(w_{2}\right)=\frac{1}{\left\langle w_{2}\right\rangle} \Phi\left(\frac{w_{2}}{\left\langle w_{2}\right\rangle}\right),
$$

where $P_{L}\left(w_{2}\right) d w_{2}$ is the probability that the width of a given configuration lies in the range $\left[w_{2}, w_{2}+d w_{2}\right]$. Alternatively, one may use the scaling form

$$
P_{L}\left(w_{2}\right)=\frac{1}{\sigma} \Psi\left(\frac{w_{2}-\left\langle w_{2}\right\rangle}{\sigma}\right),
$$

where $\sigma \equiv \sqrt{\left\langle w_{2}^{2}\right\rangle-\left\langle w_{2}\right\rangle^{2}}$ is the rms deviation of the squared width.

For KPZ in $d=1$, these scaling relations are analytically predicted and confirmed by numerical data [3]. In $d=2$, simulations of discrete models showed agreement with the scaling relation (3) [5, 13], but the shape of the scaling function was not analyzed in detail. Here, we will calculate accurate roughness distributions for three discrete models in $d=2$ : the etching model of Mello et al [14], the restricted solid-on-solid (RSOS) model of Kim and Kosterlitz 15] and a generalized RSOS model with maximum neighboring heights difference $\Delta H_{\text {max }}=2$ [15, 16], hereafter called RSOS2. Their analysis is presented in Sec. II.

Roughness distributions of the VLDS class were previously obtained by Rácz and Plischke [5], who simulated a model with Arrhenius dynamics in $d=2$. In order to analyze the shapes of the VLDS distributions, both in $d=1$ and $d=2$, we will simulate conserved solid-on-solid (CRSOS) models which show moderate finite-size effects when compared to other discrete VLDS models. Their original versions were proposed by Kim et al [17] and were rigorously proved to belong to the VLDS class [18, 19]. Their analysis is presented in Sec. III.

In Sec. IV we summarize our results and conclusions.

\section{ROUGHNESS DISTRIBUTIONS OF KPZ MODELS IN $2+1$ DIMENSIONS}

In Fig. 1 we show the distributions of KPZ models scaled according to Eq. (44), with $x \equiv\left(w_{2}-\left\langle w_{2}\right\rangle\right) / \sigma$. Results for other system sizes confirm the expected scaling of Fig. 1. The number of configurations in which $w_{2}$ was measured was near $10^{8}$ for each model and periodic boundary conditions were adopted. Eq. (3) and (4) are equivalent, but the latter was used because it provides much better data collapse for the KPZ models. 
Quantitative information on the shape of the KPZ distribution can be obtained from the dimensionless ratios of the moments of the roughness distribution $W_{n}(L) \equiv$ $\int_{0}^{\infty}\left(w_{2}-\left\langle w_{2}\right\rangle\right)^{n} P_{L}\left(w_{2}\right)$. The ratios obtained with better accuracy involve the lowest-order moments: the skewness $S \equiv \frac{W_{3}}{W_{2}{ }^{3 / 2}}$ and the kurtosis $Q \equiv \frac{W_{4}}{W_{2}{ }^{2}}-3$. Although these quantities do not characterize the whole distribution, they may be useful for the comparison with data from experiments or other model systems if data collapse methods are unreliable.

The finite-size estimates of $S$ and $Q$ for the distributions of the RSOS and the etching models are shown in Figs. 2a and 2b. Their asymptotic estimates are $S=1.70 \pm 0.02$ and $Q=5.4 \pm 0.3$. These large values show that the roughness distribution strongly deviate from a Gaussian near the maximum. For $x<x_{M}$, where $x_{M}$ is the abscissa of the maximum of the distribution in Fig. 1, the curve has an approximately Gaussian shape centered at $x_{M}$. However, for $x>x_{M}$, it significantly deviates from a Gaussian, showing a much slower decay.

The tail of the log-linear plot in Fig. 1 is not a straight line, contrary to what is observed in the 1+1-dimensional KPZ model. This is certainly related to the non-Gaussian behavior of the KPZ interface in $2+1$ dimensions [20, 21]. It is reasonable to assume that the scaling function decays as $\Psi(x) \sim x^{\beta} \exp \left(-A x^{\gamma}\right)$. Thus, for fixed $\beta$, the estimates of the exponent $\gamma$ are given by

$$
\gamma(x)=\frac{\ln \left[\frac{\ln \left(x^{-\beta} \Psi(x)\right)}{\ln \left((x-\Delta)^{-\beta} \Psi(x-\Delta)\right)}\right]}{\ln [x /(x-\Delta)]}
$$

with constant $\Delta$.

In Fig. 3 we show the effective exponents obtained from the data of the RSOS and the etching models in $L=256$ as a function of $1 / x^{2}$, for three values of $\beta$. The assumption of pure exponential decay $(\beta=0)$ leads to an asymptotic estimate $\gamma \approx 0.8$. With $\beta=-0.2$, we obtain the smallest fluctuation of $\gamma(x)$ in the range of the variable $x$ analyzed here. Results for values of $\beta$ near -0.2 give $\gamma=0.8 \pm 0.1$. Although $\beta=-1$ suggests a simple exponential decay $(\gamma=1)$ for the etching model, it shows discrepancies with the estimates for the RSOS model, reinforcing the proposal of the stretched exponential form.

One question that could be raised here is the possibility of fitting the KPZ distributions by those of $1 / f^{\alpha}$ signals 7]. However, the plots of the distributions for several values of $\alpha$ show that this procedure does not provide good fits of our data. Indeed, this can be quantitatively explained by the above values of the skewness and the kurtosis, which are 
much larger than the upper bounds for the distributions of $1 / f^{\alpha}$ signals [7]: $S=\sqrt{2}$ and $Q=3$, obtained in the limit $\alpha \rightarrow \infty$. Moreover, the simple exponential decay of the $1 / f^{\alpha}$ distributions for large $x$ disagrees with the $\exp \left(-x^{0.8}\right)$ decay of the KPZ models.

\section{ROUGHNESS DISTRIBUTIONS OF VLDS MODELS}

The CRSOS models considered here are extensions of the original ones in which the incident particle executes a random walk among neighboring columns until finding a position in which its aggregation is allowed 22]. In CRSOS1 and CRSOS2 models, the conditions $\Delta H_{\max }=1$ and $\Delta H_{\max }=2$ between neighboring columns are satisfied, respectively. Nearly $10^{8}$ configurations were used to measured $w_{2}$ in each case.

Here, we will show distributions scaled according to Eq. (3), as done in most previous works. This is also helpful for a comparison with known results for the MH theory.

In Fig. 4a we plot $\log \left[\left\langle w_{2}\right\rangle P_{L}\left(w_{2}\right)\right]$ versus $y \equiv \frac{w_{2}}{\left\langle w_{2}\right\rangle}$ for the CRSOS1 and the CRSOS2 models in $d=1$, with $L=256$. The scaling function for the MH theory, given in Ref. [4], is also shown. Its simple exponential decay contrasts with the more rapid decay of the VLDS models.

The skewness and the kurtosis of the scaled VLDS distribution are obtained along the same lines of the KPZ values: $S=1.22 \pm 0.05$ and $Q=1.6 \pm 0.2$. They contrast with the much larger values $S=1.988 \ldots$ and $Q=5.951 \ldots$ of the $\mathrm{MH}$ theory [4, 7]. An approximately $\exp \left(-y^{2}\right)$ decay is suggested by a linear fit of the data for the CRSOS1 model in the range $2 \leq y \leq 5$, as shown in Fig. 4b.

Our results in $d=2$ are shown in Fig. 4c, where scaled distributions of the CRSOS1 model $(L=64)$ and the MH theory are compared. The latter was obtained from the generating function given in Ref. [5] and the calculation of the residues of more than 20 poles closest to the origin. Both distributions have a simple exponential decay for large $y$, which is justified in the VLDS case by the linear fit shown in Fig. $5(2 \leq x<4)$. However, the VLDS distribution is much narrower than that of the MH theory: the maximum of the VLDS scaling function is $\Phi \approx 1.3$, while the maximum of the MH one is $\Phi \approx 1$ (see, e. $\mathrm{g}$. Ref. [5]), and the respective decays are, approximately, $\exp (-1.8 y)$ and $\exp (-1.3 y)$. For the VLDS distribution, the skewness and kurtosis are $S \approx 1.1$ and $Q \approx 1.8$, while the values for the MH theory are $S \approx 1.30$ and $Q \approx 2.63$. 
We also tried to fit the scaled distributions of the CRSOS1 model, in $d=1$ and $d=2$, with $1 / f^{\alpha}$-noise distributions [], with some value of $\alpha$. In $d=1$, no good fit was already expected due to the form of the decay of the scaling function discussed above. In $d=2$, there is no simultaneous agreement of the values of $S$ and $Q$ of those distributions and our estimates, for any $\alpha$, indicating that this type of fit is also impossible. In Ref. [5], a fit with $\alpha=3$ was suggested for the distribution of a growth model with Arrhenius dynamics, but this was probably a consequence of the lower accuracy of the data for that model.

\section{CONCLUSION}

Roughness distributions were calculated for several discrete models in the KPZ and the VLDS class, in one- and two-dimensional substrates, confirming the scaling relations (3) and (41). The scaling functions are different from their counterparts of the linear theories, EW (second order) and MH (fourth order). In the $2+1$-dimensional KPZ class, large values of the skewness $S$ and kurtosis $Q$ of the scaling functions are related to the high asymmetry near the maximum. For large $x$, the scaling functions decay approximately as $\exp \left(-x^{0.8}\right)$, in contrast to the simple exponential decay of the linear theories and of $1+1$-dimensional KPZ. In the 1+1-dimensional VLDS class, $S$ and $Q$ are smaller than those of the MH theory and a decay approximately as $\exp \left(-x^{2}\right)$ is obtained. For the $2+1$-dimensional VLDS class, the simple exponential decay of the scaled distribution is quantitatively different from that of the $\mathrm{MH}$ theory.

Another interesting point of our study is that none of the above distributions are fitted by those of $1 / f^{\alpha}$ noise, for any $\alpha$. It contrasts with the distributions for model and experimental interfaces in the depinning thresholds analyzed in recent works [23, 24, 25], which indicates strong effects of the non-Gaussian nature of the KPZ (in $d=2$ ) and VLDS interfaces. On the other hand, it suggests the study of other models with depinning transitions, particularly those with relatively simple dynamics whose growth phases are in the KPZ class [26], for a comparison with $1 / f^{\alpha}$ noise distributions. 


\section{Acknowledgments}

The author thanks Prof. Zoltan Rácz and Prof. Jorge de Sá Martins for helpful discussions and suggestions.

This work was partially supported by CNPq and FAPERJ (Brazilian agencies).

[1] A.-L. Barabási and H. E. Stanley, Fractal Concepts in Surface Growth (Cambridge University Press, New York, 1995).

[2] J. Krug, Adv. Phys. 46, 139 (1997).

[3] G. Foltin, K. Oerding, Z. Rácz, R. L. Workman, and R. K. P. Zia, Phys. Rev. E 50, R639 (1994).

[4] M. Plischke, Z. Rácz, and R. K. P. Zia, Phys. Rev. E 50, 3589 (1994).

[5] Z. Rácz and M. Plischke, Phys. Rev. E 50, 3530 (1994).

[6] T. Antal, M. Droz, G. Györgyi, and Z. Rácz, Phys. Rev. Lett. 87, 240601 (2001).

[7] T. Antal, M. Droz, G. Györgyi, and Z. Rácz, Phys. Rev. E 65, 046140 (2002).

[8] M. Kardar, G. Parisi and Y.-C. Zhang, Phys. Rev. Lett. 56, 889 (1986).

[9] J. Villain, J. Phys. I 1 (1991) 19.

[10] Z.-W. Lai and S. Das Sarma, Phys. Rev. Lett. 66 (1991) 2348.

[11] W. W. Mullins, J. Appl. Phys. 28 (1957) 333.

[12] C. Herring, in The Physics of Powder Metallurgy, ed. W. E. Kingston, McGraw-Hill, New York, 1951.

[13] E. Marinari, A. Pagnani, G. Parisi, and Z. Rácz, Phys. Rev. E 65, 026136 (2002).

[14] B. A. Mello, A. S. Chaves and F. A. Oliveira, Phys. Rev. E 63, 41113 (2001).

[15] J. M. Kim and J. M. Kosterlitz, Phys. Rev. Lett. 62, 2289 (1989).

[16] J. M. Kim, J. M. Kosterlitz, and T. Ala-Nissila, J. Phys. A: Math. Gen. 24, 5569 (1991).

[17] Y. Kim, D. K. Park and J. M. Kim, J. Phys. A: Math. Gen. 27, L533 (1994).

[18] Z.-F. Huang and B.-L. Gu, Phys. Rev. E 57, 4480 (1998).

[19] S.-C. Park, D. Kim and J.-M. Park, Phys. Rev. E 65, 015102 (2002).

[20] F. D. A. Aarão Reis, Phys. Rev. E 69, 021610 (2004).

[21] E. Marinari, A. Pagnani and G. Parisi, J. Phys. A 33, 8181 (2000). 
[22] F. D. A. Aarão Reis, Phys. Rev. E 70, 031607 (2004).

[23] S. Moulinet, A. Rosso, W. Krauth, and E. Rolley, Phys. Rev. E 68, 036128 (2003).

[24] S. L. A. de Queiroz, Phys. Rev. E 69, 026126 (2004);

[25] A. Rosso, W. Krauth, P. Le Doussal, J. Vannimenus, and K. J. Wiese, Phys. Rev. E 68, $036128(2003)$.

[26] F. D. A. Aarão Reis, Braz. J. Phys. 33, 501 (2003). 


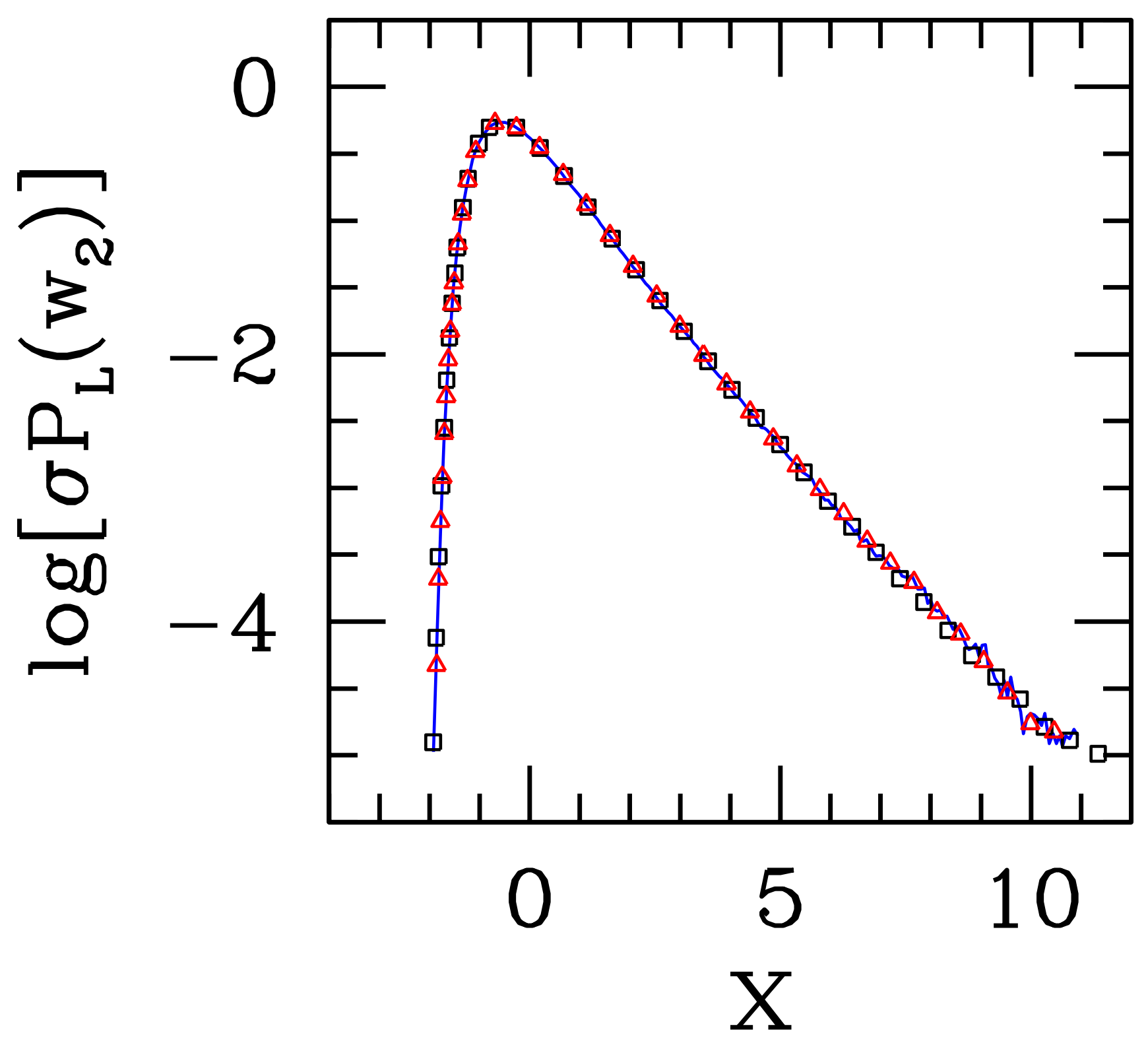

FIG. 1: Scaled roughness distributions (according to Eq. 4) at the steady states of the models: RSOS in $L=256$ (solid line), etching in $L=256$ (squares) and RSOS2 in $L=128$ (triangles). 

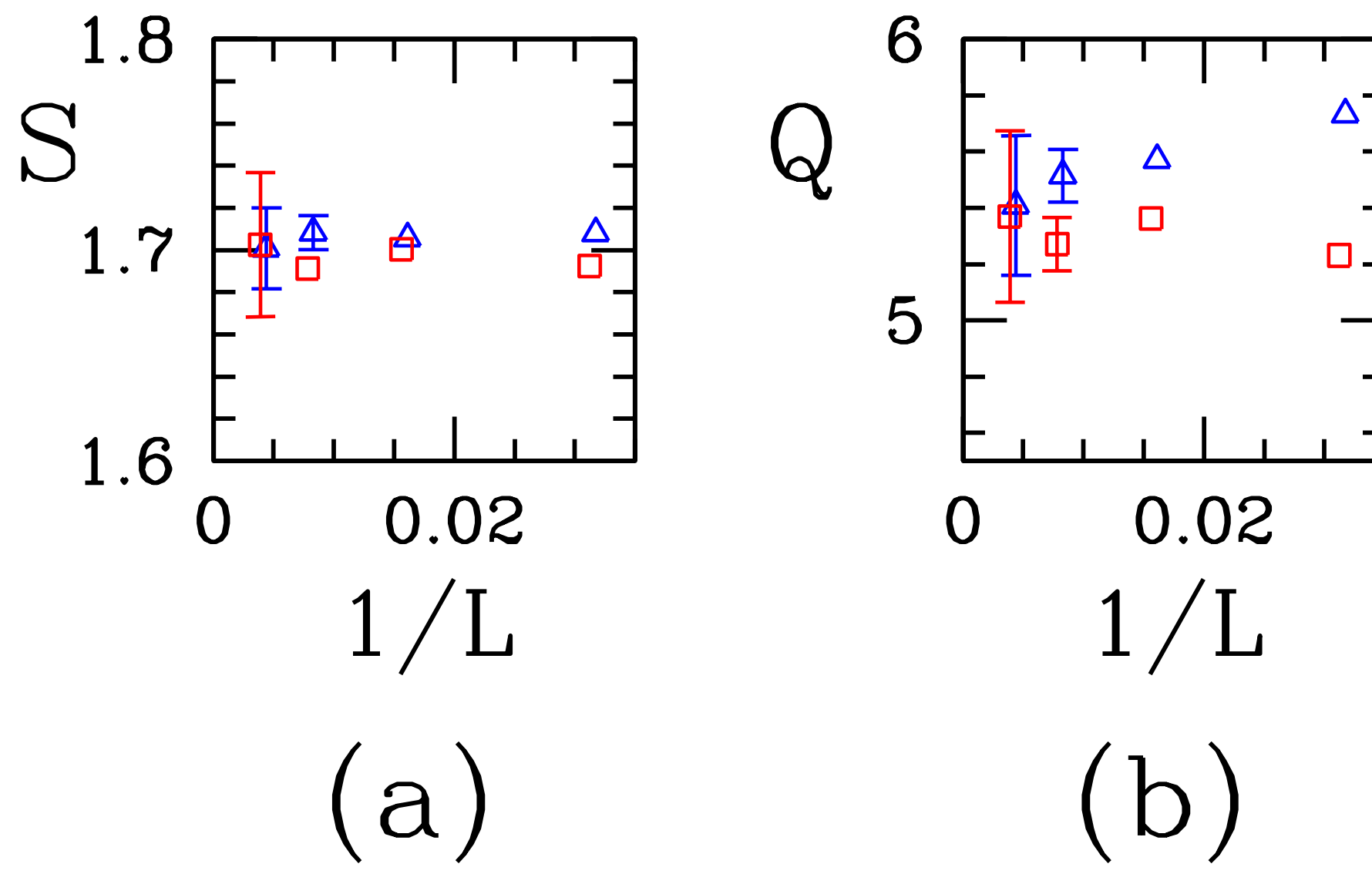

FIG. 2: (a) Skewness and (b) kurtosis of the interface width distributions at the steady states of the RSOS (squares) and etching (triangle) models as a function of inverse lattice length. 


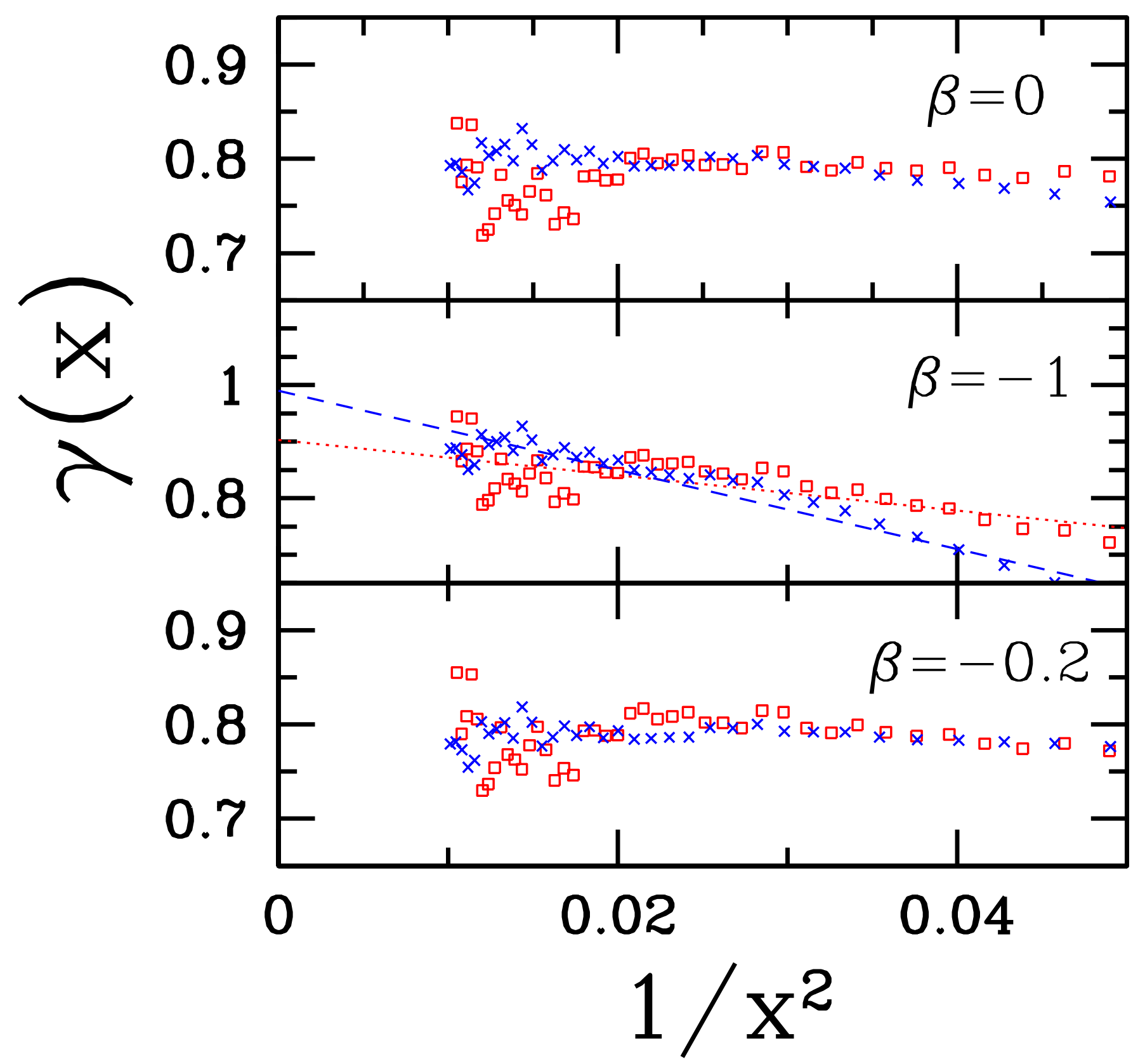

FIG. 3: Effective exponents $\gamma(x)$ of the exponential decay of the scaling functions versus $1 / x^{2}$, for three values of the exponent $\beta$ and the models RSOS (squares) and etching (crosses). The dotted (dashed) curve is a linear fit of the data of the RSOS (etching) model. 

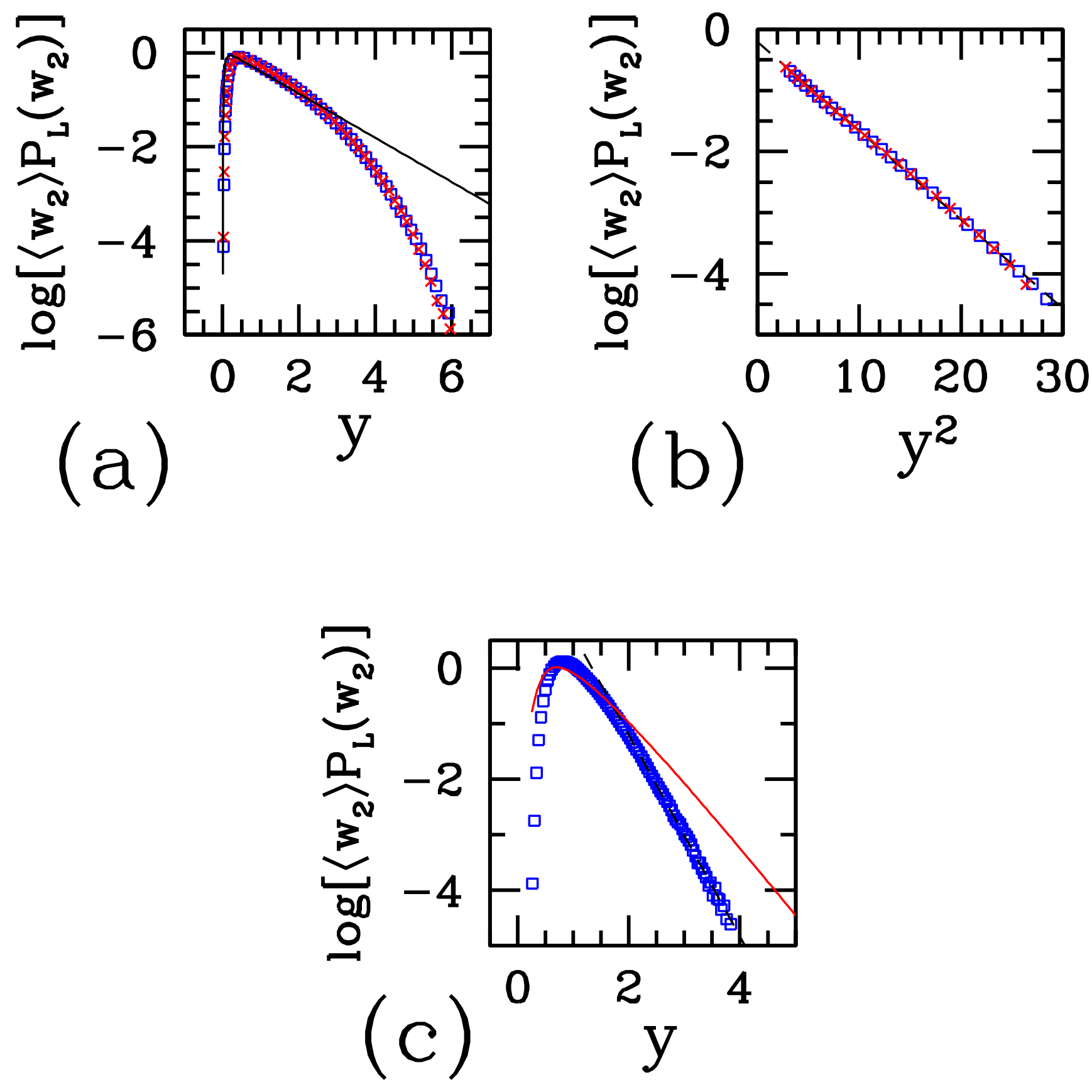

FIG. 4: (a) Scaled roughness distributions (according to Eq. 3) at the steady states of the CRSOS1 (squares) and CRSOS2 (crosses) models in $d=1$, with $L=256$. The solid curve is the scaled distribution of the MH theory in $d=1$. (b) The same distributions for CRSOS1 and CRSOS2 models as a function of $y^{2}$ and a linear fit (dashed line) of the data for the CRSOS1 model. (c) Distributions of the CRSOS1 model in $d=2,1$ ith $L=64$, and a linear fit of the data (dashed line) in the range $2<y<4$. The solid curve is the distribution of the MH theory in $d=2$. 